\title{
Characterization of a Serine Carboxypeptidase Hoscp from Holotrichia Oblita Faldermann (Coleoptera: Scarabaeoidea)
}

\author{
Dan ZHAO ${ }^{1}$, Ya-kun ZHANG ${ }^{1}$, Xiao-ping YAN ${ }^{1}$, Wei GUO ${ }^{1,2,{ }^{*}}$ and \\ Xiao-min LIU \\ ${ }^{1}$ College of Plant Protection, Agricultural University of Hebei, Baoding, Hebei, China. \\ ${ }^{2}$ Plant Science and Technology College, Beijing University of Agriculture, Beijing, \\ China.
}

${ }^{*}$ Corresponding author

Keywords: Holotrichia oblita, Serine carboxypeptidase, Gene cloning, Enzyme activity.

\begin{abstract}
In this report, we have identified a novel serine carboxypeptidase, named HoSCP, from the midgut of a destructive agricultural and landscape pest, Holotrichia oblita. HoSCP cDNA gene was cloned by immunoscreening method. Sequence analysis showed about 49kDa HoSCP consists of 457 amino acid residues with a 17-amino acid signal peptide and a conserved peptidase S10 family domain. The HoSCP protein was expressed as a recombinant protein in the yeast Pichia pastoris and enzyme activity assay showed that recombinant HoSCP had serine carboxypeptidases activity. Results of carboxypeptidase activities indicated that optimal enzyme activity occurs at $\mathrm{pH}$ 8.0, Compared with late 2nd-instar and late 3rd-instar larvae, midgut tissues of early 2nd-instar and early 3rd-instar larvae had higher levels of serine carboxypeptidase activity. The study would provide a foundation for the further research of the structure and function of HoSCP of $\mathrm{H}$. oblita.
\end{abstract}

\section{Introduction}

Carboxypeptidases are exopeptidases that remove a single amino acid residue from the C-terminus of a protein or peptide substrate. Carboxypeptidases play an important role in protein digestion in the guts of higher animals, acting to liberate free amino acids from the peptides produced by endopeptidase action, thus completing the digestive process and generating molecules that can be absorbed by the gut, via amino acid transporters[1]. Serine carboxypeptidases (SCPs) have a catalytic triad consisting of three essential amino acids, Ser, Asp, and His, which act, respectively [2]. The SCP family is organized into several clades or subfamilies (I-VI) according to their phylogenetic relationships. For instance, both type I and II SCPs have two polypeptide chains formed from a single precursor polypeptide by the excision of a linker peptide of about 50 residues[3], in contrast, type III SCPs are single-polypeptide-chain enzymes. Most SCPs have a signal sequence that directs their translocation into the secretory pathway and are believed to be vacuolar, since they are active at acidic pH[4]. Demonstration of SCPs activity in the insect midgut has been somewhat elusive, possibly due to the lack of a sensitive and specific assay system. No physiological role has yet been identified for the products of most SCP genes.

SCPs are widely distributed among fungi, plants and animals and have a range molecular weight of 40 75kDa. Currently, SCPs cDNA sequences from a few insects species have been cloned including the Haemaphysalis longicornis[5], the Sitodiplosis 
mosellana[6], and the Aedes aegypti[7]. The scarab beetle, Holotrichia oblita Faldermann (Coleoptera: Scarabaeidae), are recognized as one of the most destructive pasture pests in the northern parts of China and cause considerable loss of agriculture production and economic income. In this paper, we carried out to clone $H$. oblita serine carboxypeptidase (hoscp) gene by screening cDNA expression library of of $\mathrm{H}$. oblita larval midgut for the first time, do heterologous expression of hoscp gene, and test carboxypeptidase activity of HoSCP, which would lay a firm foundation for the researches on the biological functions of SCPs in insects and the pest biological control targeting on insects midgut.

\section{Materials and Methods}

\section{Experimental Insects and Midgut Preparation}

Adult insects were collected in the field of Baoding City, Hebei Province of China and the larvae were reared on artificial diets in entomology biochemistry and molecular pathology laboratory of Hebei Agricultural University (Baoding, Hebei). The larval midgut with its complete contents was prepared as described by Wang et al (2004)[8]. The isolated midguts were weighed individually and homogenized in $1 \mathrm{ml}$ cold deionized water with a glass tissue grinder. The homogenate was stored at $-20^{\circ} \mathrm{C}$.

\section{Cloning of Carboxypeptidase Gene by Screening cDNA Library}

A cDNA expression library of the third-instar larvae midgut of $H$. oblita was screened by subtractive immunoscreening using antibodies made against a collection of midgut PM proteins. The screening procedure was as described by the picoblue ${ }^{\mathrm{TM}}$ immunoscreening Kit (Stratagene, La Jolla, CA, USA). The clones that positive to the antibodies were cut and inserted into plasmids using Uni-ZAP XR Gigapack Clonging Kits (Stratagene, U.S.A,). The positive clones were sequenced using the dideoxynucleotide chain termination method (Sangon Co., Shanghai, China).

\section{DNA and Protein Sequence Analysis}

DNA sequence analysis and a data base search were conducted using the DNAMAN software package and NCBI BLAST. Domain analysis and protein glycosylation sites predicted using Pfam Expasy. The phylogenetic tree was constructed by the neighbor-joining method of MEGA. The serine carboxypeptidase gene cloned in this study was named hoscp.

\section{Expression of Hoscp in $\boldsymbol{P}$. pastoris}

The predicted hoscp(without signal) with a $6 \times$ His tag in C-terminal was produced by PCR using primers, which had extra bases added to include Not I (N-terminal) and EcoR I (C-terminal) restriction sites: Forward, 5'-CGGAATTCTCACTAATACCAATCGACTATTC-3'; Reverse, 5'-AAGGAAAAAAGCGGCCGCTTAATGATGATGATGATGATGTAATTCAAG AGGATTTTCTCTGCTA-3'. The amplified product was inserted into pPICK9k $P$. pastoris expression vector, and was transformed into E.coli JM109. The pPICK9k-hoscp was linearized using SalI and transformed into P. pastoris strain GS115 by electroporation. The transformed cells were spread onto YPD plates to screen high geneticin resistant colonies. To examine whether the transformants were methanol utilization phenotypes $\left(\mathrm{Mut}^{+}\right)$, a number of colonies were picked up randomly from the YPD plates and plated on both an MM plate (1.34\%yeast nitrogen base (YNB), 
$4 \times 10^{-5} \%$ biotin, $0.5 \%$ methanol] and an MD plate $\left(1.34 \%\right.$ YNB, $4 \times 10^{-5} \%$ biotin, $2 \%$ dextrose), and incubated for 3 days at $29^{\circ} \mathrm{C}$. The presence of the hoscp gene in the transformants was confirmed by PCR using yeast genomic DNA as a template with aox 1 primers carried on the vector plasmid. Selected $\mathrm{Mu}^{+}$transformant cells were grown to $\mathrm{OD}_{600 \mathrm{~nm}}$ of $5-6$ at $29^{\circ} \mathrm{C}$ in $\mathrm{BMGY}$ at $29^{\circ} \mathrm{C}$, with agitation of $250 \mathrm{rpm}$. The cells were centrifuged at $3000 \mathrm{~g}$ for $5 \mathrm{~min}$ at room temperature and resuspended in BMMYuntil an $\mathrm{OD}_{600 \mathrm{~nm}}$ of 1.0 , then the culture was grown at $29^{\circ} \mathrm{C}$ with agitation of $220 \mathrm{rpm}$. Methanol was added to a final concentration of $1 \%(\mathrm{v} / \mathrm{v})$ every $24 \mathrm{~h}$ to maintain induction. After $96 \mathrm{~h}$, the culture supernatant was collected periodically to detect for the expression of the HoSCP by western blot.

\section{Purification and the Activity of Recombinant Carboxypeptidase.}

All purification procedures were performed at $4^{\circ} \mathrm{C}$. The sample was loaded onto a $\mathrm{Ni}$-nitrilotriacetic acid (Ni-NTA) agarose column that had been equilibrated in sonication buffer. The flow rate was maintained at $0.5 \mathrm{~mL} / \mathrm{min}$. HoSCP was eluted using $15 \mathrm{mmol} / \mathrm{L}, 60 \mathrm{mmol} / \mathrm{L}$ and $500 \mathrm{mmol} / \mathrm{L}$ imidazole, respectively. Approximately $10 \mathrm{~mL}$ HoSCP was purified, fractions were analyzed by SDS-PAGE and the protein concentration was determined by the Bradford assay (Sangon of Shanghai, China). The purified HoSCP was used for enzyme activity assay as described above.

\section{Transcription Expression and Serine Carboxypeptidase Activity in Different Instar Larvae of $\boldsymbol{H}$. Oblita}

Semi-quantitative RT-PCR was carried out to assess hoscp transcriptional levels. Total RNA was isolated from different instar(from the early second to the late third instar stage) larvae midguts of $H$. oblita using the Purelink RNA Micro kit, according to the manufacturer's instructions (Invitrogen, life technologies). The mRNA abundance of hoscp gene in different instar larvae midguts was estimated by semi-quantitative RT-PCR. PCRs were carried out for 35 cycles of $45 \mathrm{~s}$ at $94^{\circ} \mathrm{C}, 30 \mathrm{~s}$ at $54^{\circ} \mathrm{C}$ and $45 \mathrm{~s}$ at $72^{\circ} \mathrm{C}$ and a final extension of $5 \mathrm{~min}$ at $72^{\circ} \mathrm{C}$. The following primers were used: $5^{\prime}-$ TTTGGCTGACACCGTTTG-3' and 5'-TTTCCGTGGTGGCATAGT-3' (101 bp) for Hoscp, 5'-ATGTTGCCATCCAAGCTGTA-3' and 5'-CCAAACGCAAAATAGCATGA-3' for actin (138 bp).

Different instar larvae of $H$. oblita from the early second to the late third instar stage were carefully dissected and used for preparing midgut extract, SCPs activity analysis as described above.

\section{Results}

\section{Identification and Sequence Analysis of the cDNA Coding for HoSCP}

By screening of the $H$. oblita midgut cDNA expression library, a cDNA was identified coding for protein with high sequence similarity to known digestive serine carboxypeptidases and named hoscp. Sequence analysis revealed that the hoscp (GenBank accession no. JF681184) was 1830bp in length, including an 78bp untranslated region at 5' end, an ORF of $1371 \mathrm{bp}$, followed by an 381bp untranslated region and a putative polyadenylation signal (AAATAA) located 14bp upstream of the polyA tail (Fig. 2). The predicted molecular weight of HoSCP preprotein was 49.4kDa. The similarity of gene sequences for hoscp with other eukaryotes indicated the presence of a conserved peptidase S10 domain which covered from $38^{\text {th }}$ to $438^{\text {th }}$ amino acid 
residues, a catalytic triad (Ser-185, Asp-361, and His-418) and an active pocket, a serine active-site motif, IAGESYAG, a histidine active-site motif, FAFLKVYGAGHMVPMDQP. Prediction of potential N-glycosylation sites using the NetNGlyc 1.0 server showed that the protein had two potential N-glycosylation sites (Fig. 1) with threonine at positions 50 and 297.

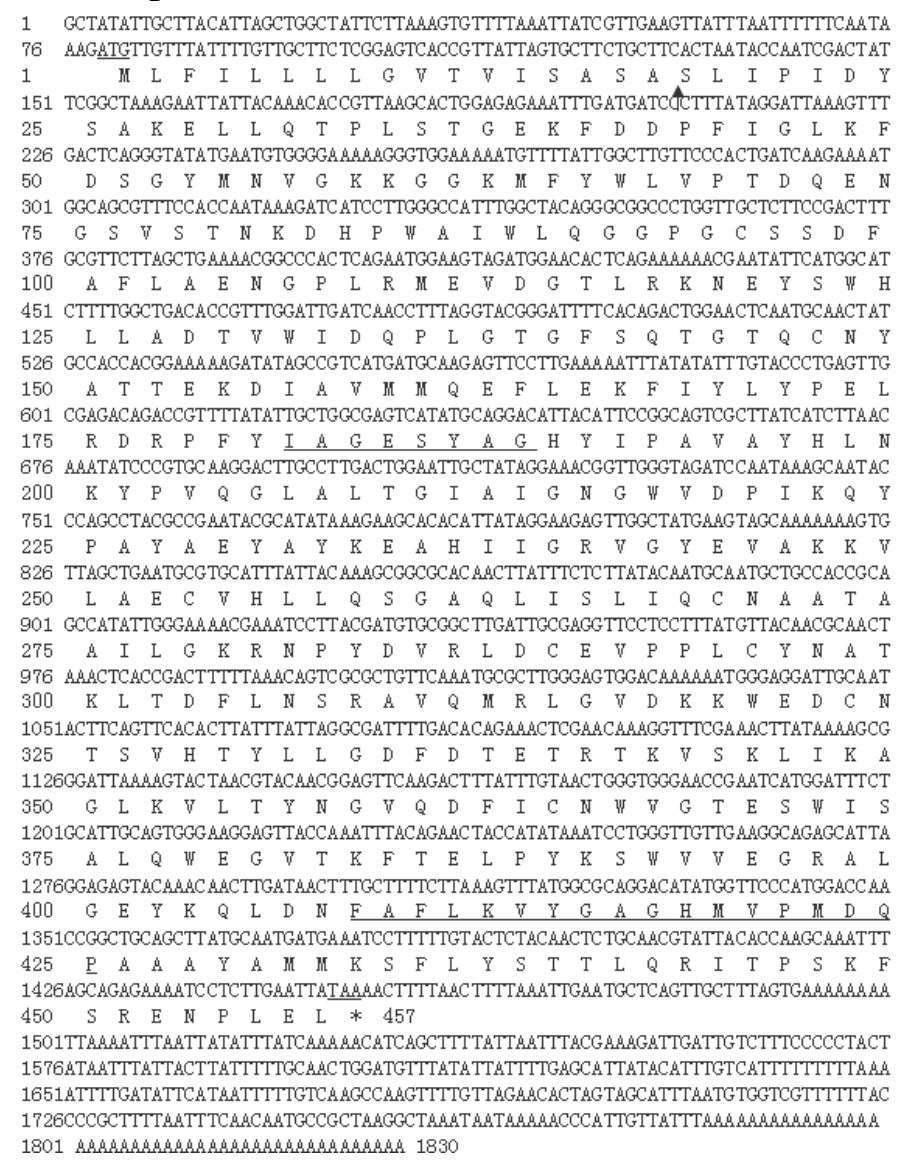

Figure 1. Nucleotide sequence and deduced amino acid sequence of the hoscp cDNA in H.oblita.

\section{Alignment of Amino Acid Sequence of HoSCP}

A search of the protein database using the National Center for Biotechnology Information revealed that HoSCP in H.oblita had sequence similarity with SCPs from several known insect. Amino acid sequence alignment showed that the catalytic triad and serine active site in SCPs were highly conserved(Fig. 2).

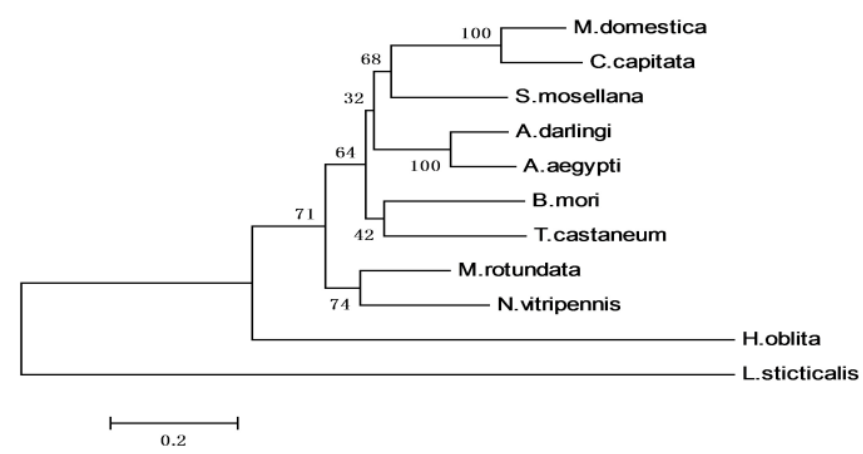

Figure 2. Phylogenetic analysis of HoSCP in H. oblita and several known SCPs in insects. 


\section{Expression of $\mathrm{HoSCP}$ in $\boldsymbol{P}$. pastoris}

Auxotrophic GS115 strains could not grow normally on the MD and MM plates for lack of histidine, however transformants with recombinant plasmid pPICK9k-hoscp was regained the property of histidine synthesis and didn't require histidine for growth. Phenotype of transformants was identified on MM and MMH plates (Fig. 3A). PCR was conducted to confirm the presence of the hoscp gene in the transformants by using yeast genomic DNA as a template. The result showed that two bands of approximately $2.2 \mathrm{~kb}$ corresponding to the $A O X 1$ gene and $1.8 \mathrm{~kb}$ corresponding to the hoscp gene were detected on agarose gel of the transformants containing recombinant plasmid pPICK9k-hoscp, while the negative control strains containing plasmid pPICK9k only revealed a $0.5 \mathrm{~kb}$ band corresponding to the flanking sequence of $A O X 1$ gene (Fig. $3 \mathrm{~B}$ ).

A

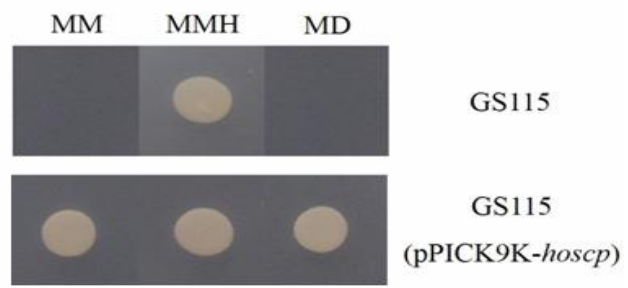

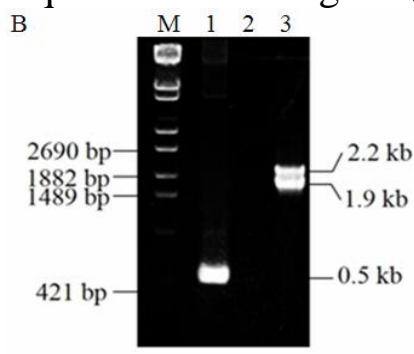

Figure 3. Identification of the phenotype of the recombinant GS115 strains.

(A) Phenotype identification of the recombinant GS115 strains. Auxotrophic GS115 strains could not grow on the MD and MM plates for lack of histidine, however transformants with recombinant plasmid pPICK9k-hoscp was regained the property of histidine synthesis and didn't require histidine for growth. (B) Molecular identification of recombinant GS115 strains. Lane M correspond to DNA molecular weight standards of $\lambda E c o T-14$. Lane 1 correspond to the negative control of strains containing plasmid pPICK9k. Lane 2 correspond to the negative control of deionized water as a template. Lane 2 correspond to the transformants containing recombinant plasmid pPICK9k-hoscp.

P.pastoris GS115/ pPICK9k-hoscp transformants were cultivated in Buffered Glycerol-complex Medium (BMGY) for the HoSCP production. After 96h of methanol induction, the culture supernatants were collected periodically to detect for the expression by western blot analysis using the anti-His Tag antibody and a band of approximately $52 \mathrm{kDa}$ was detected constantly (Fig. 4).

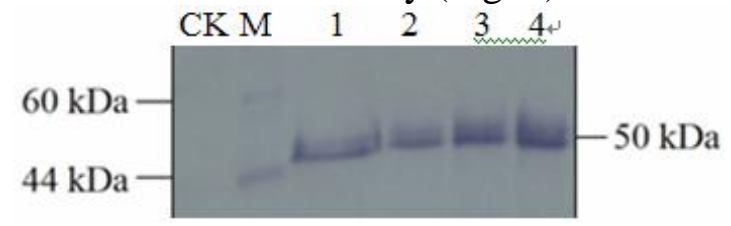

Figure 4. Western blot analysis of the HoSCP expressed in recombinant GS115 strains.

CK serves as a negative control of protein expression of GS115 strains containing pPIC9K. Lane M correspond to the protein molecular weight standards. Lanes 1-4 correspond to the of recombinant protein expression at $29^{\circ} \mathrm{C}$ with methanol induction after $24,48,72,96$ hours, respectively.

\section{Enzyme Activities of HoSCP expressed in P. pastoris}

After $72 \mathrm{~h}$ of methanol induction, the culture supernatants were collected and used for enzyme activity assay. The specific activity of the crude enzyme solution was determined to be $0.011 \mathrm{U} / \mathrm{ml}$ (Fig. 5A, B). 
A

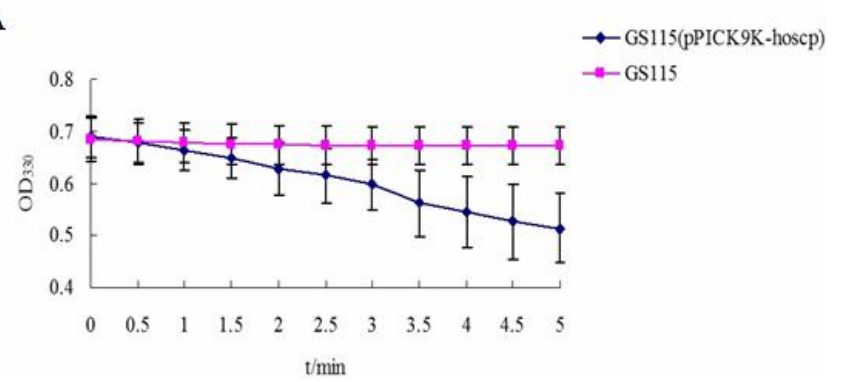

B

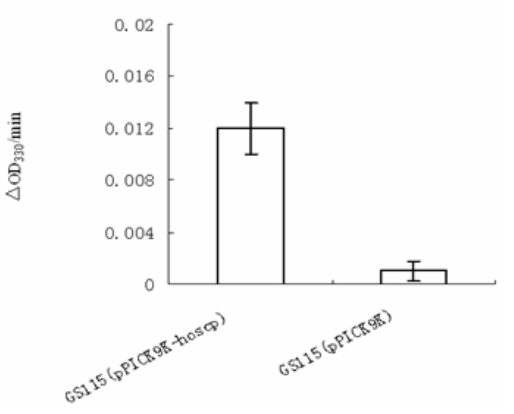

Figure 5. Carboxypeptidase activities of HoSCP expressed in P. pastoris.

Carboxypeptidase activity was determined using the substrate FAAK. (A) The activity determination curve of HoSCP expressed in recombinant $P$. pastoris GS115 strain; (B) Serine carboxypeptidase relative activities of supernatant of recombinant and host $P$. pastoris GS115 strain, points in the graph are the means from three replicated determinations and error bars indicate the $95 \%$ confidence intervals of the means

\section{Transcriptional Level and Enzyme Activity of HoSCP in Different Instar Larvae Midguts of $\boldsymbol{H}$. oblita}

The transcriptional levels of $H o S C P$ was examined by semi-quantitative RT-PCR using specific primers on RNA extracted from different instar larvae midgets of $H$. oblita(Fig. 6A). HoSCP gene had higher expression levels in the early 2nd-instar and early-3rd instar larvae. As shown by enzyme activity analysis, midgut tissues of early 2nd-instar and early 3rd-instar larvae had higher levels of serine carboxypeptidase activity then the late 2nd-instar and late 3rd-instar larvae per milligram of midgut, respectively, which had approximately two times level of activity (Fig. 6B), which indicated that the HoSCP in midgut was upregulated after larval moult.

A
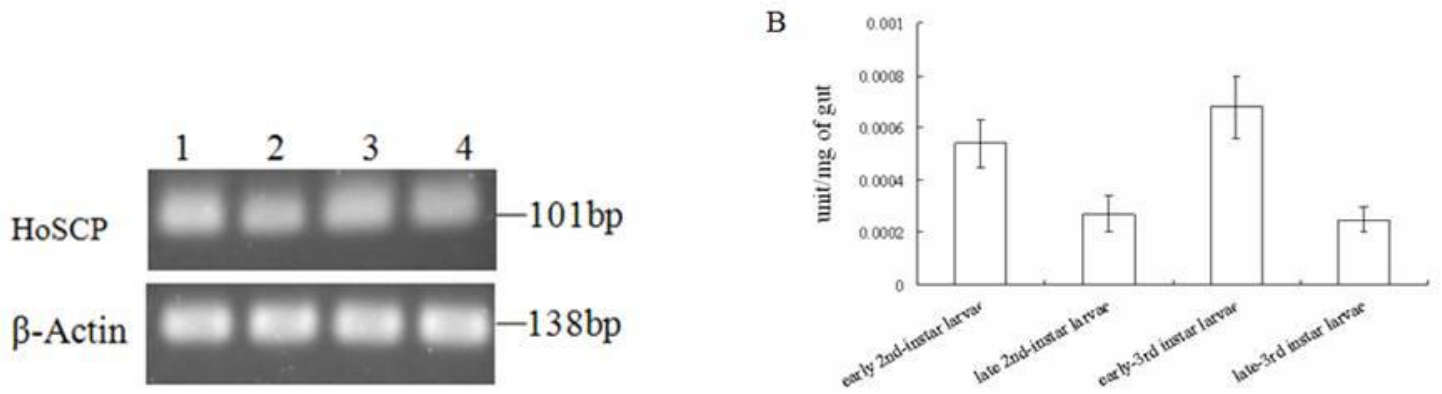

Figure 6. HoSCP transcriptional analysis in different tissues.

(A) Semi-quantitative RT-PCR analysis of HoSCP at different instar larvae midguts.1: early 2nd-instar larvae; 2: late 2nd-instar larvae; 3: early-3rd instar larvae; 4: late 3rd-instar larvae. (B) Midgut serine carboxypeptidase activities from different instar larva of $H$. oblita. Enzyme activities were determined from five larvae and the means from the five determinations are shown in the graphs. Error bars indicate the $95 \%$ confidence intervals of the means. Midgut carboxypeptidase activities are shown as unit/mg of midgut.

\section{Discussion}

SCPs are widely distributed among fungi, plants and animals. In insects, however, SCP sequences and their physiological roles have been identified from only a few insects species. A serine carboxypeptidase (HISCP1) identified in the midgut of the hard tick Haemaphysalis longicornis is strongly expressed in the midgut and is supposed to localize at lysosomal vacuoles and on the surface of epithelial cells. Its expression is upregulated during the course of blood-feeding, which imply that HISCP1 may function as a lysosomal protease in the process of blood digestion[5]. In our study, we provide 
the first report of a serine carboxypeptidase in the midgut of $H$. oblita larvae. The HoSCP protein had the presence of a conserved of peptidase S10 domain consisting of a catalytic triad (Ser-185, Asp-361, and His-418) and an active pocket, a serine active-site motif, IAGESYAG, a histidine active-site motif, FAFLKVYGAGHMVPMDQP. Prosite protein pattern search predicted that the protein had two potential N-glycosylation sites with threonine at positions 50 and 297. Although HoSCP possessed the conserved regions and catalytic triad of the SCPs, its overall sequence similarity with other SCPs was $<50 \%$. Phylogenetic analysis demonstrated that the homology of HoSCP with SCP from T. thermophila was higher than other species, whose intestinal function remained unknown.

Expression of the HoSCP as a recombinant protein in P.pastoris had allowed its functional properties to be partly characterized. It was speculated that HoSCP expressed in $P$. pastoris GS115 was a precursor enzyme with several proteolytic cleavage sites and activation of the proenzyme by proteases was necessary for its activity. Both type I and II SCPs have two polypeptide chains formed from a single precursor polypeptide by the excision of a linker peptide. In the case of cathepsin A, a SCP in humans, a $54 \mathrm{kDa}$ precursor was cleaved into a mature heterodimer of 32 and $20 \mathrm{kDa}$ subunits, which are linked by disulfide bonds[9,10], and a 34 and $20 \mathrm{kDa}$ form also exists as a transient processing intermediate[11]. Type III SCPs are single-polypeptide-chain enzymes. As no proteolytic cleavage site except for the signal peptide site was detected at the amino acid sequence level in HISCP1 in H.longicornis, which indicating that HISCP1 was a single-chain enzyme, like CPY[5]. Protease digestive experiments are necessary for determination of which SCPs type the HoSCP belonging to.

The intestinal $\mathrm{pH}$ of midgut was very alkaline $(\mathrm{pH}$ 8.0 9.5) in larvae of the Scaraboidea[12, 13, 14, 15] The optimal $\mathrm{pH}$ range for the hydrolysis of FAAK by $H$. oblita larval midgut extract was at $\mathrm{pH}$ 8.0, which was not consistent with the SCPs activity at acidic $\mathrm{pH}$. HISCP1 showed optimum enzyme activity at acidic $\mathrm{pH}$ and had a substrate preference for Z-Phe-Leu, properties which are consistent with those of cathepsin $\mathrm{A}[5,10]$. This result was similar to the situation of cysteine proteinases in beetles (Coleoptera: Chrysomeloidae). The match between optimal and actual alkaline $\mathrm{pH}$ of the midgut to be weaker in four species of the cerambycids. These findings suggested that either a close correlation between midgut $\mathrm{pH}$ and the proteolytic $\mathrm{pH}$ optimum is not necessary for adequate digestive efficiency, or that midgut $\mathrm{pH}$ is a more constrained digestive feature and there has been insufficient time for it to shift upwards to maximize serine proteinase activity[14]. The phenomenon may be either partly as the presence of carboxypeptidase $\mathrm{B}$ activites whose activities at alkaline $\mathrm{pHs}$ [8] or that the FAAK was not the optimum substrate for SCPs assay of $H$. oblita larval midgut. A various of substrates would be prepared for the determination of HoSCP activity preference in further study. SCPs activities in different instar larva of $H$. oblita changed constantly. Midgut extracts from early 2 nd-instar and early-3rd instar larvae had higher levels of SCPs activity than those of late 2nd-instar and late-3rd instar larvae respectively, which had approximately half the level of activity. More food intake and vigorous metabolism would contribute to the higher levels of SCPs in early 2nd-instar and early-3rd instar larvae. Furthermore, it was speculated that expression of SCPs in midgut was upregulated after feeding food as HISCP1 in H. Longicornis[5].

\section{Acknowledgement}

This research was financially supported by the earmarked fund for Modern 
Agro-industry Technology Research System, China; the Natural Science Foundation of Hebei Province (C2016204082).

\section{References}

[1] Bown, D.P., Gatehouse, J.A. Characterization of a digestive carboxypeptidase from the insect pest corn earworm (Helicoverpa armigera) with novel specificity towards C-terminal glutamate residues, Eur J Biochem. 271(2004) 2000-2011.

[2] Bendtsen, J.D., Nielsen, H., Von Heijne, G. , Brunak, S. Improved prediction of signal peptides: SignalP 3.0, J Mol Biol. 340(2004) 783-795.

[3] Doan, N.P. , Fincher, G.B. The A-and B-chains of carboxypeptidase I from germinated barley originate from a single precursor polypeptide, J Biol Chem. 263(1988) 11106-11110.

[4] Bienert, M.D., Delannoy, M., Navarre, C., Boutry, M. NtSCP1 from tobacco is an extracellular serine carboxypeptidase III that has an impact on cell elongation. Plant Physiol. 158(2012) 1220-1229.

[5] Motobu, M., Tsuji, N., Miyoshi, T., Huang, X., Islam, M.K., Alim, M.A. , Fujisaki, K. Molecular characterization of a blood-induced serine carboxypeptidase from the ixodid tick Haemaphysalis longicornis. FEBS J. 274(2007) 3299-3312.

[6] Mittapalli, O., Wise, I.L. , Shukle, R.H. Characterization of a serine carboxypeptidase in the salivary glands and fat body of the orange wheat blossom midge, Sitodiplosis mosellana (Diptera: Cecidomyiidae). Insect Biochem Mol Biol. 36(2006) 154-160.

[7] Cho, W.L., Deitsch, K.W., Raikhel, A.S. An extraovarian protein accumulated in mosquito oocytes is a carboxypeptidase activated in embryos. Proc Natl Acad Sci USA. 88(1991) 10821-10824.

[8] Wang, P., Li, G. , Kain, W. Characterization and cDNA cloning of midgut carboxypeptidases from Trichoplusia ni. Insect Biochem Mol Biol. 34(2004) 831-843.

[9] Galjart, N.J., Gillemans, N., Meijer, D. , D'azzo, A. Mouse "protective protein". cDNA cloning, sequence comparison, and expression. J Biol Chem. 265(1990) 4678-4684.

[10] Hiraiwa, M., Saitoh, M., Arai, N., Shiraishi, T., Odani, S., Uda, Y., Ono, T. , O'brien, J.S. Protective protein in the bovine lysosomal beta-galactosidase complex. Biochim Biophys Acta. 1341(1997) 189-199.

[11] Bonten, E.J., Galjart, N.J., Willemsen, R., Usmany, M., Vlak, J.M. , D'azzo, A. Lysosomal protective protein/cathepsin A. Role of the "linker" domain in catalytic activation. J Biol Chem. 270(1995) 26441-26445.

[12] Broadway, R.M. , Villani, M.G. Does host range influence susceptibility of herbivorous insects to novel plant proteinase inhibitors? Entomol. Exp. Applic. 76 (1995) 303-313. 
[13] Christeller, J.T., Shaw, B.D., Gardiner, S.E. , Dymock, J. Partial purification and characterization of the major midgut proteases of grass grub larvae(Costelytra zealandica, Coleoptera: Scarabaeidae). Insect Biochem. 19(1989) 221-231.

[14] Johnson, K.S., Rabosky, D. Phylogenetic distribution of cysteine proteinases in beetles: evidence for an evolutionary shift to an alkaline digestive strategy in Cerambycidae. Comp Biochem Physiol B Biochem Mol Biol. 126(2000) 609-619.

[15] Wolfson, J.L., Murdock, L.L. Diversity in digestive proteinase activity among insects. J. Chem. Ecol. 16(1990) 1089-1102. 